\title{
Cancer, Infection and Disturbances of The Integrity of Tissue Homeostasis: The Most Significant Triggers for Molecular Mimicry and Autoimmunity in Dermatology?
}

\author{
Georgi Tchernev · Torello Lotti · José-Carlos Cardoso · Nobuo Kanazawa · Claudio Guarneri · \\ Uwe Wollina
}

Published online: 6 May 2014

(C) Springer-Verlag Wien 2014

Molecular Mimicry (MM) is a modern useful concept to try to understand the etiology, pathogenesis, treatment and prevention of autoimmune disorders of the skin and beyond the skin [1].

MM cannot be better defined than the possibility that sequence similarities between self-peptides and foreign molecules are sufficient to start a valid crossactivation of human B and/or T cells [2]. Nevertheless, lack of amino acid identity between the tissue and the de novo-generated tumour antigens does not exclude

\section{G. Tchernev $(\bowtie)$}

Medical Faculty, University Hospital Lozenetz,

Koziak street 1,

1407 Sofia, Bulgaria

e-mail: georgi_tchernev@yahoo.de

\section{G. Tchernev}

Policlinic for Dermatology and Venerology, Saint Kliment Ohridski

University,

Sofia, Bulgaria

Prof. Dr. T. Lotti, MD, MD (Hon)

University of Rome "G. Marconi”,

Rome, Italy

\section{J.-C. Cardoso, MD, PhD}

Dermatology Department, University Hospital of Coimbra, Coimbra, Portugal

N. Kanazawa, MD, PhD

Department of Dermatology, Wakayama Medical University, Wakayama, Japan

C. Guarneri, MD

Dept. of Social Territorial Medicine, Section of Dermatology, University of Messina,

Messina, Italy

U. Wollina, MD

Department of Dermatology \& Allergology, Hospital Dresden-

Friedrichstadt,

Dresden, Germany the phenomenon of molecular mimicry as the major generator of sarcoidosis [3]. There is growing evidence of the mimicry phenomena, caused not only by the similarity between the amino acids but also between the elements which connect segments in the immunological cascade and which may also be affected by external factors [3].

Such activation, induced by pathogen-derived peptides, can result in acute, subacute or (more often) chronic skin disease of autoimmune nature upon "peptide mimic" activation via specific T cell receptors which generates a self-maintaining circle based on a cross reaction with self epitopes [2].

Molecular mimicry (MM) is one of the most enigmatic medical phenomena with crucial importance for the generation of autoimmunity in dermatology [1]. The fact that there are no tests capable of projecting an objective image of molecular mimicry should not lead us to ignore or undervalue its presence in medicine and dermatology in particular [4].

In general terms, the basic generators of molecular or antigen mimicry can be classified in three major groups:

1. Bacteria, viruses and parasites with the capacity of initiating an autoimmune response directed towards various tissue structures. When these structures are located in the skin they can give rise to autoimmune skin diseases like bullous dermatoses, lichen planus and lichenoid reactions, sarcoidosis and the sarcoidlike reactions, and likely many others.

2. Tumor antigens, which are also capable of initiating a cross-reaction that may be associated with some of the above-mentioned dermatoses.

3. Drugs and other exogenous substances deposited into the human body, which in one way or another may contribute to skin disease via disruption of the homeostatic integrity of the tissue. 
The fact that these three generators of molecular mimicry may lead to similar clinical conditions, requiring fundamentally different diagnostic and therapeutic approach, is of undeniable interest [4].

The degree to which these clinical conditions relate to autonomous diseases, or rather to a single type of reaction, is a matter of debate [4]. Unfortunately there are currently no specific genetic features and panels that could be widely employed in the process of differentiating sarcoidosis from sarcoid-like reactions, lichen planus from lichenoid tissue reactions and idiopathic bullous dermatoses from their counterparts associated with an underlying condition or drugs [4].

According to some data in the literature, certain genetic abnormalities are characteristic of one or another skin disease but in spite of this, such markers have not been widely used in the standard diagnostic procedures and have not even been embedded in the so called inclusion criteria of the respective disease [4]. During the last 15 years we have witnessed the absence of the so-called criteria update and in view of the rate at which genetics and immunology are currently developing, this seems to be an unthinkable and an unexplained observation [5].

In medicine, establishing the difference between certain idiopathic diseases and their counterparts associated with an underlying cause is a serious problem [4]. Oversimplification, blending of these two notions, or placing them under a common denominator may lead to undesired consequences for the affected individuals [4]. Such consequences may be due to the fact that sarcoid, lichenoid and bullous dermatoses can be triggered by de novo appearing tumor antigens, and, in addition, treatment may be initiated without excluding such triggering factors. Implementing such an immunosuppressive therapy in the setting of an underlying neoplasm may lead to progression of the underlying tumor, with the undesirable consequences that it may have for the overall prognosis [4].

We believe that the lack of knowledge regarding genetic signatures, regular update and inclusion criteria relating to certain autoimmune diseases, as well as the lack of specific models of the clinical behavior of immunological diseases (sarcoidosis, bullous and lichenoid dermatoses, for instance), are the presumed reasons for the varying course of the same disease after apply- ing similar therapeutic strategies to different patients, with outcomes that may vary from improvement to no response or even progression of the disease $[4,5]$.

The understanding of the control of molecular mimicry is and will be a major issue in our discipline. Presently, we envision that it can be reached mainly by inducing tolerance to the host auto-antigen via proper vaccination.

Unfortunately the very popular treatments with immune suppressive drugs including cyclosporine, azathioprine, methotrexate, and biologics become ineffective because cells and tissues have already been destroyed after the onset of the infection.

A new era based on a policy of proper vaccination strategy is starting now for the Dermato-Venereologist dealing with auto-immune skin diseases.

\section{Conflict of interest}

No conflict of interest.

No financial disclosure.

\section{References}

1. Guarneri F, Guarneri C. Molecular mimicry in cutaneous autoimmune diseases. World J Dermatol. 2013 Nov 2;2(4):36-43.

2. Tchernev G, Ananiev J, Cardoso JC, Wollina U, Verma SB, Patterson JW, Dourmishev LA, Tronnier M, Okamoto H, Mizuno K, Kanazawa N, Gulubova M, Manolova I, Salaro C. Sarcoidosis and molecular mimicry-important etiopathogenetic aspects: current state and future directions. Wien Klin Wochenschr. 2012 Apr;124(7-8):227-38.

3. Tchernev G, Wollina U. Sarcoidosis, cancer and molecular mimicry. Int J Immunopathol Pharmacol. 2013 Jul-Sep;26(3):753-5.

4. Tchernev G, Tana C, Schiavone C, Cardoso JC, Ananiev J, Wollina U. Sarcoidosis vs. Sarcoid-like reactions: The Two Sides of the same Coin? Wien Med Wochenschr. 2014 Mar 25; [Epub ahead of print].

5. Hunninghake GW, Costabel U, Ando M, Baughman R, Cordier JF, du Bois R, Eklund A, Kitaichi M, Lynch J, Rizzato G, Rose C, Selroos O, Semenzato G, Sharma OP. ATS/ERS/ WASOG statement on sarcoidosis. American Thoracic Society/European Respiratory Society/World Association of Sarcoidosis and other Granulomatous Disorders. Sarcoidosis Vasc Diffuse Lung Dis. 1999 Sep;16(2):149-73. 